\title{
América Latina a la luz del poder económico mundial de China: el caso de Brasil y Ecuador y el comportamiento de su política exterior. Estudio comparativo
}

\author{
Lorena Herrera-Vinelli \\ Doctorante en Estudios Internacionales \\ FLACSO-Ecuador \\ Recibido: 13 de junio de 2016 \\ Aceptado: 12 de septiembre de 2016
}

\section{Resumen}

El presente artículo tiene como objetivo delinear los factores que inciden en el afianzamiento de las relaciones sino-latinoamericanas. Se sostiene que el interés político, comercial y financiero de América Latina respecto a China se explica en función del posicionamiento logrado por dicho país como segunda economía mundial. El análisis de los casos de Brasil, como un país relativamente industrializado y Ecuador como un

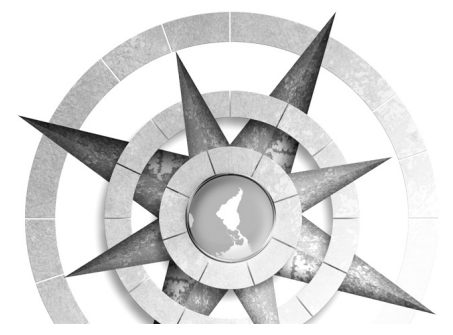

país pequeño, sirve como un referente empírico para comprender y explicar cómo se ha comportado la política exterior de ambos países en torno a China y permite indagar sobre los ejes prioritarios de su relación desde una perspectiva comparada. El artículo concluye que si bien ambos países tienen una relación estratégica con China, el caso de Brasil muestra matices de una política exterior fuertemente vinculada a China en las esferas políticas, comerciales y financieras, mientras que en el caso de Ecuador la dependencia financiera subyace como elemento de peso en la política exterior.

Palabras clave: economía de América Latina, comercio exterior, política exterior, China, Ecuador, Brasil, Temas de Nuestra América 


\section{Abstract}

This article aims to outline the factors that influence the strengthening of Sino-Latin American relations. It is argued that the political, commercial and financial interest of Latin America towards China is explained in terms of the positioning achieved by that country as the second world's economy. The analysis of the cases of Brazil as a relatively industrialized country and Ecuador as a small country serve as an empirical reference to explain and understand how the foreign policy of both countries has behaved with respect to China, and it allows inquiring about the priority axes of their relationship throughout a comparative methodology. The article concludes that although both countries have a strategic relationship with China, the Brazilian case reveals a strongly linked foreign policy to China in political, trade and financial spheres, whereas Ecuador's financial dependence underlies like the most important element of its foreign policy.

Keywords: Latin America economy, international trade, foreign policy, China, Ecuador, Brazil, Temas de Nuestra América

\section{Resumo}

O objetivo de este artigo é descrever os fatores que afetam o fortalecimento das relações sino-latino-americanas. Se argumentado que o interesse político, comercial e financeiro na América Latina em relação a China é explicado em termos de posicionamento alcançado por esse país como a segunda economia mundial. A análise dos casos do Brasil como um país relativamente industrializado e Equador como um país pequeno serve como um referente empírico para entender e explicar como se comportou a política externa de ambos os países em torno da China e, ainda, permitir obter informações sobre as prioridades da sua relação, a partir de uma perspectiva comparativa. $\mathrm{O}$ artigo conclui que, embora ambos os países têm uma relação estratégica com a China, o caso brasileiro mostra nuances de uma política externa fortemente ligada a China nos âmbitos políticos, comerciais e financeiros, ao passo que no caso do Equador, a dependência financeira é o elemento mais importante na política externa.

Palavras chave: Economia da América Latina, comércio exterior, política externa, China, Equador, Brasil, Questões de Nossa América 


\section{Introducción}

Desde el ascenso de China como la segunda economía mundial, sus esfuerzos políticos y diplomáticos se han centrado en el planteamiento de una agenda común con la región latinoamericana. Ello, incluye una agenda de política exterior conocida como el Libro Blanco de las Políticas de China hacia América Latina 2008, basado fundamentalmente en la cooperación, el beneficio recíproco y la ganancia compartida. La profundización de relaciones sino-latinoamericanas está fuertemente influenciada por intereses geopolíticos que se visibilizan en la demanda de recursos energéticos, de productos alimentarios provenientes del sector agrícola y en la penetración de productos manufacturados chinos en los mercados de la región. Sin embargo, diversos países han afianzado sus relaciones con China en distintas esferas. En el ámbito comercial, China constituyó el principal destino de exportaciones de Brasil y Chile representando el 43,9\% del valor total de las exportaciones de ambos países en 2013 y el 61,8 \% de Brasil, Chile y Perú para el año 2014. Por otro lado, China representó el 35,3\% de las importaciones de Brasil y Chile en el año 2013 versus un $60 \%$ en materia de importaciones de Brasil, Chile y Perú en el año 2014, mientras que 17 países de la región importaron productos provenientes de China; de éstos, Argentina, Brasil, Chile, Cuba, Ecuador, Panamá, Perú y Venezuela presentaron un porcentaje superior al $10 \%$ del total de sus importaciones que en su mayoría responden a productos manufacturados en 2013 (The World Factbook, 2013). Adicionalmente, China se ha consolidado como el principal financista internacional de la región, desplazando a las fuentes tradicionales de financiamiento como el Banco Mundial, especialmente en los casos de Venezuela, Argentina y Ecuador; estos países destinan los créditos otorgados por China al financiamiento de sus economías. En este marco, resulta necesario contextualizar los principales matices de China como segunda economía mundial, para luego esbozar las líneas generales de su relación con América Latina.

\section{China en el actual escenario económico mundial}

China se ha consolidado como la segunda economía mundial en el año 2011 y primera potencia comercial en el año 2014, logrando uno de los procesos de modernización económica más dinámicos de la historia en el nivel internacional (Rodríguez, 2015: p. 339). Sin embargo, para comprender el poder económico mundial alcanzado por el gigante asiático, resulta necesario referirnos al periodo que marcó el inicio de 
la etapa de China como economía de mercado. En este sentido, Deng Xiaoping $^{29}$ instauró una serie de reformas económicas en China conocidas como el modelo "open doors" que a través de la liberalización económica buscaba el salto de China de una economía cerrada marcada por el aislacionismo a una economía de mercado. Las relaciones internacionales de China bajo el liderazgo de Deng incluyeron una estrategia basada en la diplomacia de la apertura y el reconocimiento diplomático en oposición a Taiwán (Rodríguez, 2015: p. 346). Junto con la reforma económica de Deng en la década de los setenta y el fin de la guerra fría, China logró dar pasos significativos para integrarse en la economía global. A pesar de que no era miembro formal del Acuerdo General de Tarifas y Comercio (GATT), pudo beneficiarse de las políticas de liberalización de comercio de países como Estados Unidos a través de la cláusula de la nación más favorecida (Ripley, 2002, p.134). Su ingreso a la Organización Mundial del Comercio en el año 2001, le permitió además afianzar un crecimiento económico sin precedentes.

29 Previo a convertirse en el máximo líder de la República Popular China, Deng Xiaoping fue uno de los principales miembros del Partido Comunista Chino liderado por Mao Zedong, en donde, junto con otros líderes, fue acusado de traidor por formar parte de un ala de derecha dentro del partido que buscaba insertar ideas capitalistas en el modelo comunista instaurado por Mao Zedong en China.
De hecho, el comercio exterior ha sido el principal instrumento de política exterior con lo cual China ha logrado un crecimiento económico sin precedentes que según Slipak entre 1978 y 2011 alcanzó un promedio de $10 \%$ anual (2014: p. 12). En consecuencia, datos actuales reflejan que las exportaciones chinas alcanzaron un crecimiento económico entre el 2005 y 2010 de 107,70 $\%$, mientras que en el 2010 y 2013 lograron un crecimiento del $40 \%$. Cabe señalar, que en el año 2013 China logró un crecimiento económico del $7 \%$, lo cual repercutió positivamente en la recuperación del crecimiento mundial (CEPAL, 2014: p. 15) ${ }^{30}$. El significativo ritmo de sus exportaciones siguieron creciendo con 2342 miles de md fueron exportados al mundo en 2014 (Estadísticas del Comercio Internacional, 2015, p.44).

En términos del crecimiento económico que alcanzaron las exportaciones chinas, entre el 2005 y 2010 éstas ascendieron a $107,70 \%$, mientras que en el 2010 y 2013 lograron un crecimiento del $40 \%$. Es así que en el 2013, China ocupó el primer lugar como exportador de equipos de oficina y telecomunicaciones con una cifra

30 Porcentajes de crecimiento calculados a partir de la información disponible en UNCTADstat que indica que el comercio de exportaciones de mercancías de China (en millones de dólares) en adelante 'md', en el 2005 se sitúa en 761953 md; en 2010 asciende a 1577754 md; y para el 2013 aumenta a $2209007 \mathrm{md}$. 
de 594 md. y el mismo año se convierte en el primer exportador mundial de textiles y vestidos alcanzando los $285 \mathrm{md}$. que representa el $39 \%$ en el sector vestido y el $35 \%$ en textiles. (Estadísticas del Comercio Internacional, 2014: p. 54-60).

La tasa de crecimiento de sus exportaciones comerciales fue de $6.0 \%$ en 2014, posicionándolo como la primera potencia exportadora del mundo y el crecimiento de su producto interno bruto fue de $7.3 \%$ el mismo año (Conferencia de Naciones Unidas sobre Comercio y Desarrollo, 2016), siendo uno de los países con mayor crecimiento de este indicador en el nivel internacional. Del total de sus exportaciones que se situaron en 2.24 trillones de dólares en el 2014, las mismas están distribuidas parcialmente entre sus principales socios y destinos comerciales como Estados Unidos (16.9 \%), Hong Kong (15.5\%), Japón (6.4 \%), Corea del Sur (4.3\%) entre los cuales cabe señalar, no se encuentra ningún país latinoamericano (The World Factbook, 2015). En cuanto al crecimiento de sus importaciones, entre el 2005 y 2010 alcanzaron 111,56 \%, y entre el 2010 y 2013 se situaron en un 39,65 \%; en este último año, China registra un superávit en su balanza comercial de 259015 md. $^{31}$

31 Porcentajes de crecimiento calculados a partir de la información disponible en UNCTADstat que indica que las importaciones de China para
Cabe señalar, que el posicionamiento económico que China ha logrado afianzar en el nivel internacional le ha servido como plataforma para aumentar su grado de influencia política en varias esferas regionales como es el caso de la Asociación de Naciones del Sudeste Asiático (ASEAN), el Foro de Cooperación Económica Asia-Pacífico (APEC), el bloque BRICS, y recientemente inició diálogos regionales con América Latina a través de la I Cumbre CELAC-China 2014, que según Rodríguez ha sido la principal iniciativa diplomática en el afianzamiento político de las relaciones con América Latina (2015: p.110).

\section{El libro blanco chino 2008: instrumento político y diplomático en sus relaciones con América Latina}

Las relaciones políticas y diplomáticas de China se han centrado en el planteamiento de una agenda común con la región latinoamericana. Ello, incluye una agenda de política exterior conocida como el Libro Blanco de las Políticas de China hacia América Latina 2008. Los pilares del Libro Blanco 2008, se encuentran estrechamente relacionados con los cinco principios de coexistencia pacífica

el 2005 se sitúan en 659953 md; para el 2010 ascienden a $1396247 \mathrm{md}$ y en el 2013 llegan a 1 949992 md. 
sobre los cuales se basan las relaciones internacionales de China respecto de la región. Como sostiene Xiaoping: "China siempre se basa en los cinco principios de coexistencia pacífica para establecer relaciones con terceros Estados. Con cualquier país, sea de ideología izquierda o derecha, siempre que respete los cinco principios de coexistencia pacífica ${ }^{32}$, China desarrollará relaciones normales de Estado" (Xiaoping, 2015: p. 73). Por otro lado, el Libro Blanco chino deja en claro que, la política exterior de dicho país hacia la región incluye una estrategia de desarrollo basada en ganar-ganar y no en una estrategia ganar-perder o juego de suma cero. Esta afirmación se sustenta en el estudio de Rodríguez quien argumenta que la estrategia de China en América Latina difiere de la implementada por las potencias tradicionales a lo largo de la historia, en la que se encuentra inmersa la categoría de enemigo (Rodríguez, 2015: p. 348). Por tanto, a través de este instrumento político-diplomático, China ha delimitado como principales ámbitos de interés a los recursos naturales que posee la región.

32 Los cinco principios de coexistencia pacífica son: 1) integridad territorial y soberanía 2) no agresión; 3) no interferencia en asuntos internos 4) igualdad y mutuo beneficio y 5) coexistencia pacífica.

\section{China y América Latina: ¿intereses comunes estratégicos?}

En su relacionamiento con América Latina, China se ha configurado en el escenario internacional como un actor estratégico con intereses asentados en distintos ejes. En el contexto de crecimiento económico que ha logrado afianzar China en más de tres décadas, se evidencia la necesidad de fortalecer el comercio internacional con América Latina, región que está en capacidad de ofertar productos alimentarios pero también energéticos (Slipak, 2014: p. 12). Su modelo de industrialización a gran escala demanda importantes recursos energéticos, muchos de los cuales se encuentran en las zonas con la mayor biodiversidad del planeta al interior de América Latina. De hecho, la demanda de China por fuentes energéticas de la región ha aumentado notablemente con algunos países latinoamericanos productores de petróleo. Ello, se complementa con la demanda de otras fuentes energéticas como gas, carbón, cobre, etc. Algunos estudios sostienen que la demanda de los commodities que ejerce China sobre la región, ha reprimarizado las economías de la misma, por su reorientación hacia actividades de tipo primarias extractivas o maquilas, con nulo valor agregado, a lo que se suma un grave impacto sobre la tenencia de tierras, recursos y territorios,

112 América Latina a la luz del poder económico mundial de China: el caso de Brasil y Ecuador y el comportamiento de su política exterior. Estudio comparativo

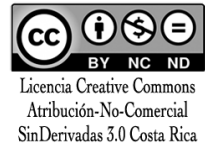
Lorena Herrera-Vinelli 
refiriéndose a las actividades extractivas a cargo de China en diversos países de América Latina (Svampa, 2013: p. 3).

Según Ramírez, el marcado interés comercial de China por los commodities que posee América Latina se explicaría en parte, por el déficit comercial que China atravesó desde finales de los noventa en diversos sectores para la industrialización de cobre, mineral de hierro, níquel y la soja (2013: p. 241). Por su parte, Slipak sostiene que la mayoría de las exportaciones de la región latinoamericana a China se produce en una relación asimétrica, dado que exportan productos provenientes de actividades primario-extractivas o de productos industriales basados en recursos naturales, mientras que las importaciones de América Latina respecto a China se encuentran sumamente diversificadas y constan de productos con alto con- tenido de valor agregado (2014: p. 7). Pese a ello, y como señala Ramírez: "el comercio sino-latinoamericano ha situado a China como el socio comercial más dinámico, desplazado a Japón como primer socio asiático en la región, y augurado el inminente desplazamiento de la Unión Europea como segundo socio comercial en la zona para 2016" (2013: p. 241). De esta manera, en lo que se refiere al ámbito comercial los países que sitúan a China como su primer destino comercial en materia de exportaciones en el año 2014 son: Brasil, Chile y Perú mientras que países como Belice, Costa Rica, República Dominicana, Ecuador, El Salvador, Guatemala, Haití, Honduras y Nicaragua no tienen a China en el ranking de sus cinco principales destinos comerciales (The World Factbook, 2014). 


\section{Gráfico 1.}

\section{Principales porcentajes de exportación de América Latina hacia China en 2014}

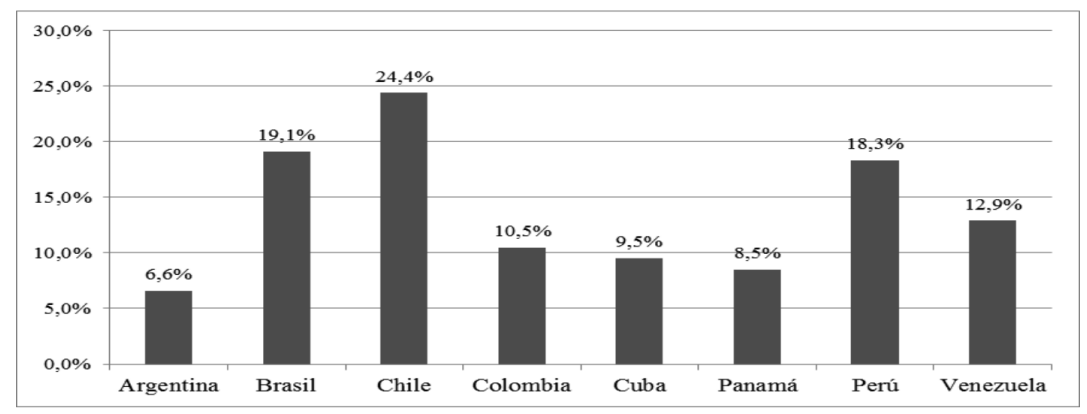

Fuente: Elaboración propia a partir de datos contenidos en The World Factbook (2015).

Por su parte, a continuación se muestran los porcentajes de importación de América Latina respecto a China en 2014:

\section{Gráfico 2.}

\section{Porcentajes de importación de América Latina respecto a China en 2014}

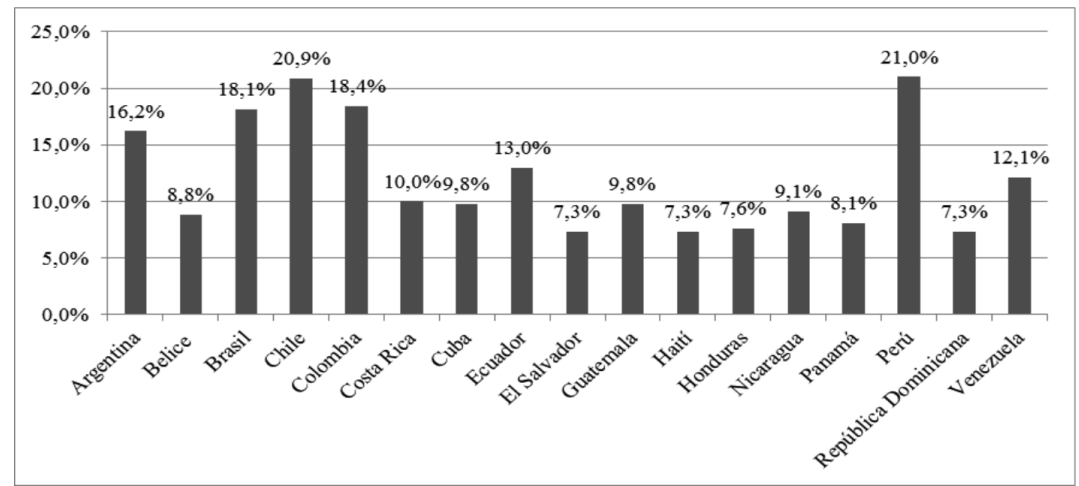

Fuente: Elaboración propia a partir de datos contenidos en The World Factbook (2015).

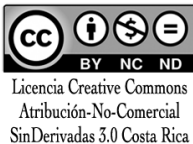

114 América Latina a la luz del poder económico mundial de China: el caso de Brasil y Ecuador y el comportamiento de su 
De otro lado, China se ha convertido en el principal financista internacional de América Latina. Según "China-Latin America Finance Database 2015" del Centro de Estudios Diálogo Interamericano de Washington "Desde 2005, el Banco de Desarrollo de China y el Banco de Exportaciones-Importaciones de China han proporcionado 125 mil millones de dólares en compromisos de préstamos a América Latina y el Caribe (ALC) y las empresas de propiedad estatal" (Gallagher y Myers, 2014). El financiamiento recibido por varios de los países latinoamericanos se concentra en áreas como la infraestructura, la minería, y proyectos energéticos, y que se vinculan con las necesidades y demandas establecidas por cada gobierno de conformidad con su modelo de desarrollo (Gallagher y Myers, 2014), lo que en palabras de Ramírez se explica como "el viraje centro izquierda de gobiernos latinoamericanos" (2013: p. 241).

\section{Gráfico 3.}

\section{Financiamiento chino a América Latina por país (en millones de dólares) 2005-2015}

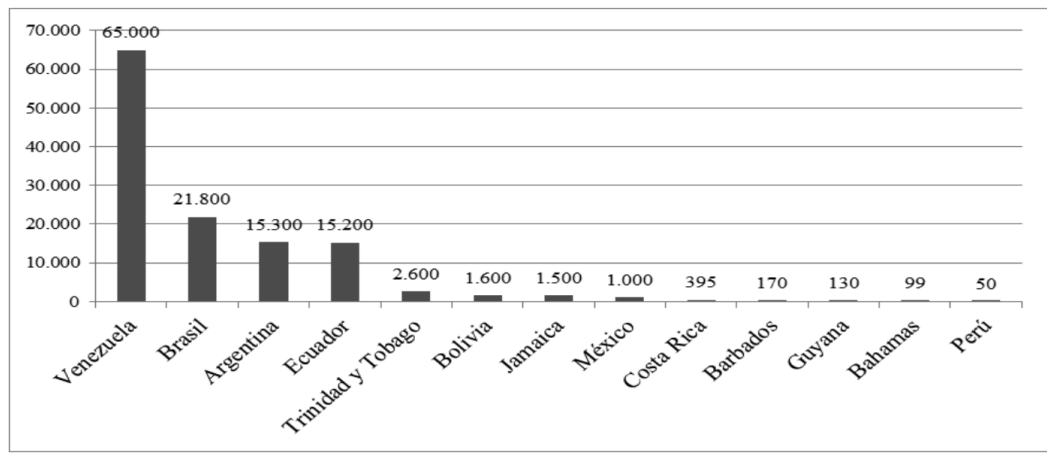

Fuente: Elaboración propia a partir de Gallagher y Myers (2014). http://www.thedialogue.org/map_list/

Es interesante conocer que algunos paí- Mundial. Este comportamiento lo obses latinoamericanos han optado por servamos en el caso de Venezuela con fuentes de financiamiento chinas en un $82,52 \%$ de créditos chinos versus lugar de aquellas provenientes de or- $0 \%$ de créditos contraídos con el Banganismos occidentales como el Banco co Mundial; Argentina con 41,90 \% de 
créditos chinos versus 24,81\% con el Banco Mundial; y Ecuador con un 73,54\% de créditos chinos versus 1,55 $\%$ con el Banco Mundial, en el periodo 2005-2011 (Basabe y Martínez, 2014, p.149). Dicho esto, a continuación resulta interesante profundizar sobre el comportamiento de la política exterior de Brasil como país relativamente industrializado y Ecuador como país pequeño en su relación con China.

\section{El comportamiento de la política exterior de Brasil: miradas multinivel en su relación con China}

Desde el mandato presidencial de Lula, se amplía el interés por generar alianzas estratégicas con China. Como candidato presidencial en el año 2002, Lula mostró los matices de sus principales propuestas en materia de política exterior, especialmente vinculada a temas como la conformación de una agenda propia en materia económica y de desarrollo, a través de un modelo alternativo, con énfasis en reducir los riesgos y la vulnerabilidad de Brasil en los mercados financieros internacionales, la ampliación del espectro comercial, y con severas críticas frente al intervencionismo en la política interna de organismos internacionales como el Fondo Monetario Internacional y el Banco Mundial, entre los principales (Grasa, 2004: p. 8). "Ya en el propio programa, aparecían otros rasgos llamados a tener especial protagonismo, la aproximación a países de importancia regional como Sudáfrica, India, China y Rusia" (Grasa, 2004: p. 8). Lula se pronunció además en favor de generar una alianza estratégica con Pekín la cual podría incluir apoyo en las organizaciones internacionales así como una colaboración científica permanente (Spitaels, 2009: p. 276).

En este sentido, la política exterior de Brasil durante los dos mandatos de Lula 2003-2010, denominada como la autonomía por la diversificación, implicó un etapa en la que Brasil contaba tanto con los recursos cuanto con capacidades para emprender una diversificación de sus relaciones (Pereyra, 2013: p.109-114). De esta manera, como parte de esta estrategia, China pasó de ser un actor relevante para los intereses de Brasil en el discurso de Lula a convertirse en un socio estratégico durante su mandato. Mansilla señala que "China se convirtió en el primer socio comercial de Brasil, posición anteriormente ocupada por EUA. De este modo, "Beijing absorbió el 13,2\% de las exportaciones brasileiras así como originó el 12,5\% de las importaciones del país suramericano" (2012, p.2). El afianzamiento de las relaciones comerciales de Brasil con

116 América Latina a la luz del poder económico mundial de China: el caso de Brasil y Ecuador y el comportamiento de su 
China se explicaría principalmente por su necesidad de ampliar su inserción en el mercado mundial, lo cual consistía en uno de los mayores intereses de su agenda de política exterior. Es decir, consolidarse como una especie de "global trader" como lo define Duarte y Trindade en el cual "el globalismo o "universalidad de la acción diplomática" intenta hallar una forma concreta de actuación para cumplir con el objetivo de convertirse en un "global trader" (2008: p. 90).

Como señaló el ex presidente Lula: "A partir del año pasado 2003, nuestro gobierno tomó la decisión estratégica de estrechar cada vez más nuestras relaciones con China" (Duarte y Trindade, 2008, pp.92 citando a Silva, 2004). Este acercamiento se visibilizó posteriormente en las cifras del intercambio comercial de ambos países que entre el periodo 2000 y 2001 alcanzaron un crecimiento de $72,72 \%$, mientras que en el periodo 2002 y 2003 se situaron en el $80 \%$. $^{33}$

Para el año 2004, el ex presidente Lula realizó una de las visitas más importantes a China, en la que las empresas de Brasil cerraron aproximadamente 15 acuerdos comerciales con el gigante asiático en diversas áreas tales como siderúrgica, petróleo, por ejemplo la firma del acuerdo entre Petrobras y Sinopec para comercialización, exploración, producción, refinación, construcción de ductos petrolíferos; telecomunicaciones, termoeléctricas, inversiones, entre los más importantes. En esta visita, el ex presidente Lula declaró: "Compartimos el mismo objetivo de integrar nuestras economías, de forma competitiva, al mercado globalizado del Siglo XXI" (El Universo, 2004). Los acuerdos generados dinamizaron las relaciones políticas y comerciales de ambos países en distintos ámbitos. Un tema de especial trascendencia, es que durante el mandato del ex presidente Lula, Brasil junto con Rusia, India y China, conformaron el bloque BRICS en mayo de 2008. Sudáfrica, se unió en el año 2011. De hecho, China representa la mitad del tamaño del PIB combinado de los cuatro países denominados BRICS (Rosales y Kuwayama, 2012: p. 15). Con Dilma Rousseff, las relaciones entre ambos países continuaron el rumbo iniciado por su predecesor. El alcance de las relaciones con China durante su mandato se puede visibilizar a través del crecimiento del comercio internacional alcanzado por Brasil, principalmente en materia de exportaciones como se expone a continuación.

33 Los porcentajes han sido calculados a partir de la información disponible en el artículo de Duarte y Trindade (2008).

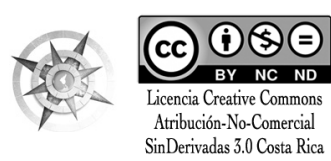




\section{Comercio exterior y financiamiento internacional}

El comercio internacional de exportación de mercancías de Brasil entre el año 2005 y 2010 alcanzó un crecimiento de $70,35 \%$, y entre el 2010 y 2013 se situó en $19,94 \%{ }^{34}$. La estructura de exportaciones de mercancías por grupo de productos se encuentra distribuida de la siguiente manera en 2016: 36 \% alimentos; $4 \%$ materia prima agrícola; $16 \%$ minerales y metales; $9 \%$ combustibles; $34 \%$ productos manufacturados; $1 \%$ otros. (Conferencia de Naciones Unidas sobre Comercio y Desarrollo, 2016).

Para el año 2014, China constituyó el principal mercado de exportación de mercancías de Brasil con un porcentaje del 19,1\%, mientras que Estados Unidos fue su segundo socio comercial con el $12,6 \%$, y en tercer lugar Argentina con el 6,8\% (The World Factbook, 2014). En cuanto a las importaciones de mercancías entre el 2005 y 2010 Brasil alcanzó un crecimiento de $146,73 \%$, y entre el 2010 y 2013 sus im-

34 Porcentajes de crecimiento calculados a partir de la información disponible en UNCTADstat que indica que el comercio internacional de exportación de mercancías de Brasil para el año 2005 se sitúa en 118529 md; para el año 2010 alcanza la cifra de 201915 md, mientras que para el año 2013 asciende a $242179 \mathrm{md}$. portaciones asciendieron a $30.75 \% .^{35}$ De manera similar al sector de exportaciones, China constituyó el principal socio comercial de Brasil en cuanto a importaciones con el $15,6 \%$ para el año 2013 y el $18,1 \%$ en el año 2014 (The World Factbook, 2013-2014). Los principales productos de exportación de Brasil a China están conformados por soja, minerales de hierro, petróleo crudo, animales, azúcares, tabaco, algodón, cueros, cobre refinado, entre los más importantes y que presentaron una reducción importante en el 2014, principalmente por la caída de los precios internacionales de los commodities como es el caso de la soja y los minerales de hierro (Informe del Consejo Empresarial Brasil-China, 2015: p. 4).

Adicionalmente, China no sólo tiene una importancia significativa para Brasil en cuanto al comercio de mercancías, sino que también ocupa un espacio trascendente en materia de los créditos internacionales a los que ha accedido para el financiamiento de distintos proyectos. Brasil se ha convertido en el segundo mayor deudor de China en el nivel de América Latina con un monto que asciende a 21.8 millones de dólares,

35 Porcentajes de crecimiento calculados a partir de la información disponible en UNCTADstat que indica que las importaciones de mercancías en el año 2005 alcanzaron los 77628 md; en 2010 los 191537 md y en el 2013 se situaron en 250447 md.

118 América Latina a la luz del poder económico mundial de China: el caso de Brasil y Ecuador y el comportamiento de su política exterior. Estudio comparativo

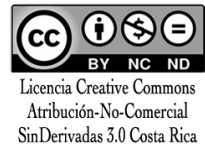




\section{Tabla 1.}

Principales productos exportados a China (millones de dólares)2013-2014

\begin{tabular}{|lcc|}
\hline \multicolumn{1}{c}{ Descripción } & 2013 & 2014 \\
\hline Soja, incluso triturada & 17148 & 16615 \\
\hline Minerales de hierro y sus concentrados & 15933 & 12303 \\
\hline Petróleo crudo & 4035 & 3473 \\
Celulosa & 1347 & 1424 \\
\hline Azúcares & 1432 & 880 \\
\hline Cueros y píeles sin preparar & 429 & 554 \\
\hline Aves de corral & 441 & 519 \\
\hline Ferro aleaciones & 497 & 504 \\
\hline Aceite de soja & 517 & 340 \\
\hline Tabaco no manufacturado & 454 & 334 \\
\hline Algodón & 189 & 333 \\
\hline Pasta química de madera & 230 & 282 \\
\hline Cueros preparados & 210 & 271 \\
\hline Cobre refinado y aleaciones de cobre & 587 & 256 \\
\hline Otros & 2577 & 2529 \\
\hline Total & 46026 & 40617
\end{tabular}

Fuente: Elaboración propia a partir de datos contenidos en el Informe del Consejo Empresarial de Brasil-China.http://www.cebc.org.br/sites/default/files/china-brazil_update_v.final_oficial.pdf

y que desde el año 2005 hasta el año llo de China, la empresa SINOPEC, 2015 se contabilizarían en ocho crédi- China Ex Im Bank, Banco de China tos internacionales aproximadamente. y la empresa ICBC, en sectores como Los mismos han sido adquiridos con minería, energía, infraestructura y otros entidades como el Banco de Desarro- (Gallagher y Myers, 2014). 


\section{El comportamiento de la política exterior de Ecuador: miradas multinivel en su relación con China}

Ecuador, un país pequeño andino, es uno de los países que ha fortalecido sus relaciones exteriores con China como consecuencia del modelo de desarrollo que ha perseguido el gobierno del Presidente Rafael Correa. Según el Plan Nacional del Buen Vivir (2013-2017) se intenta superar las lógicas tradicionales basadas en la acumulación de capital y el crecimiento económico; y en su lugar, se apunta al desarrollo humano como objetivo fundamental para combatir la pobreza. La transformación de la política internacional sin duda fue uno de los grandes ejes del programa de gobierno de Correa. Ella, se encuentra reflejada en diversos documentos, siendo uno de ellos el Manifiesto Ideológico del Movimiento Alianza PAIS $^{36}$, mismo en el cual se declara el interés por la integración latinoamericana, la cooperación y la solidaridad como un objetivo estratégico (2006, p. 12). En este contexto, se vislumbra el gran interés por la cooperación sursur, reorientando así la política exterior hacia aquellos países o grupos de países que han sido catalogados como periféricos o del tercer mundo, siendo denominados como "las grandes víctimas

36 Organización política que preside y representa a Rafael Correa. del sistema capitalista hegemónico al desempeñar un rol subalterno y dependiente" (Manifiesto Ideológico Alianza PAIS, 2006: p. 12). Por su parte, el artículo 416 de la Constitución literal 10 determina la necesidad de promover un orden global multipolar con la participación activa de bloques económicos y políticos regionales y el fortalecimiento de las relaciones horizontales.

Vale señalar que, la nueva dinámica en la que se inserta la política internacional del Ecuador en el mundo, fue replicada tanto en el Plan Nacional de Desarrollo (2007-2010) como en el Plan Nacional (2010-2013). No obstante, es el Plan Nacional del Buen Vivir (2013. 2017) el que delinea la importancia de China para el gobierno de Correa, donde se refiere a la crisis del capitalismo y su repercusión en la desaceleración del poder económico de Estados Unidos como uno de los factores que explican la necesidad por replantear las relaciones comerciales, políticas y económicas del Ecuador en el nivel internacional (2013, p. 13).

En este nuevo orden económico mundial, Ecuador apuesta por consolidar sus relaciones económicas de manera prioritaria con actores estratégicos en el sistema mundial, y en este marco China es considerado un actor clave para Ecuador como se plasma a continuación: 
"En este renovado contexto mundial, emergen dos fenómenos que favorecen directamente al posicionamiento del Ecuador y de América Latina en el sistema-mundo: la emergencia de procesos de integración política y económica regional, y el incremento de los vínculos políticos y comerciales con nuevos actores globales" (Plan Nacional del Buen Vivir, 2013: p. 13). En este marco, la política exterior de Ecuador durante el mandato de Correa ha priorizado la relación con China, en buena medida, por los intereses que ha perseguido su gobierno en el nivel doméstico e internacional.

\section{Comercio exterior y financiamiento internacional}

El comercio de mercancías de Ecuador en términos de sus exportaciones totales entre el año 2005 y 2010 se situaron en $73,16 \%$, mientras que entre el 2010 y 2013 alcanzaron un crecimiento de $42,65 \%{ }^{37}$. En el caso de las importaciones de mercancías entre el 2005 y 2010 alcanzaron un crecimiento porcentual de $100 \%$ y en el periodo 2010 y 2013 se situaron en el 32,60\%. ${ }^{38} \mathrm{La}$

37 Porcentajes de crecimiento calculados a partir de la información disponible en UNCTADstat que indica que las exportaciones de Ecuador para el año 2005 se situaron en $10100 \mathrm{md}$; en el 2010 alcanzaron la cifra de $17490 \mathrm{md}$, mientras que para el año 2013 ascendieron a $24951 \mathrm{md}$.

38 Porcentajes de crecimiento calculados a partir de la información disponible en UNCTADstat que indica que en el caso de las importaciones de estructura de exportaciones por grupo de producto se encuentra distribuida de la siguiente manera: combustibles (52\%), productos alimentarios (34\%), productos manufacturados $(6 \%)$ y productos agrícolas (4\%) (Conferencia de Naciones Unidas sobre Comercio y Desarrollo, 2016).

En el periodo enero-abril 2013, China se situó entre los principales destinos de las exportaciones no petroleras de Ecuador, ocupando el décimo segundo lugar con un monto total de exportaciones que asciende a los 82493 miles de dólares (FOB) (Boletín Mensual de Comercio Exterior del Ecuador, 2013: p.11), mientras que de enero a octubre de 2015, China ocupó el cuarto destino de las exportaciones no petroleras del Ecuador con una participación del $4.90 \%$ del total exportado (Boletín Mensual de Comercio Exterior 2015: 11). Entre los principales productos de exportación no petrolera a China se destacan: harina de pescado, desperdicios de desecho de cobre, camarones, langostinos, madera aserrada, bananas frescas, minerales de cobre, oro y concentrados, entre otros.

mercancías se observa que en el año 2005 la cifra corresponde a $10287 \mathrm{md}$, para el año $2010 \mathrm{se}$ duplica y alcanza los $20591 \mathrm{md}$, mientras que para el 2013 se mantiene en los 27305 md. SinDerivadas 3.0 Costa Rica 
En enero-abril 2014 la cifra ascendió a portaciones ecuatorianas enero-abril, su punto más alto con 106786 miles 2012-2014).

de dólares (FOB) (Evolución de las ex-

\section{Tabla 2.}

Principales productos exportados a China (miles de dólares)

$$
\text { Enero-abril 2012-2014 }
$$

\begin{tabular}{|c|c|c|c|c|}
\hline Descripción & Unidades & 2012 & 2013 & 2014 \\
\hline Harina de pescado & $\mathrm{FOB}^{39}$ & 4421 & 24682 & 9990 \\
\hline Desperdicios y desechos de cobre & $\mathrm{FOB}$ & 15569 & 14549 & 11612 \\
\hline $\begin{array}{l}\text { Los demás camarones, } \\
\text { langostinos y demás decápodos } \\
\text { no congelados }\end{array}$ & FOB & 5662 & 9582 & 16905 \\
\hline Maderas aserradas o desbastadas & $\mathrm{FOB}$ & 2993 & 4194 & 6305 \\
\hline Bananas frescas & $\mathrm{FOB}$ & 2228 & 4041 & 23666 \\
\hline $\begin{array}{l}\text { Minerales de cobre y sus } \\
\text { concentrados }\end{array}$ & FOB & 0 & 3304 & 3624 \\
\hline $\begin{array}{l}\text { Minerales de oro y sus } \\
\text { concentrados }\end{array}$ & $\mathrm{FOB}$ & 429 & 2860 & 9455 \\
\hline Otros & $\mathrm{FOB}$ & 16557 & 16994 & 25228 \\
\hline Total & FOB & 47589 & 80206 & 106786 \\
\hline
\end{tabular}

Fuente: Elaboración propia a partir de datos contenidos en el Informe Evolución de las exportaciones ecuatorianas" Enero/abril (2012-2014). Disponible en:

http://www.proecuador.gob.ec/wpcontent/uploads/2013/07/PROECUADOR_IC_03-31.pdf

39 Significa Free on Board. FOB es un incoterm que se utiliza en el comercio exterior, y que se refiere al precio que tiene una determinada mercancía cuando la misma es colocada en el buque del comprador en el puerto de embarque del país de origen.

122 América Latina a la luz del poder económico mundial de China: el caso de Brasil y Ecuador y el comportamiento de su política exterior. Estudio comparativo 
De otro lado, China se ha convertido en la principal fuente de financiamiento del Ecuador. Durante el mandato del presidente Rafael Correa, el país ha adquirido una significativa deuda internacional que asciende a $15.2 \mathrm{mi}$ llones de dólares, ocupando de esta manera el cuarto lugar como deudor de China en el nivel de América Latina desde el año 2009 hasta el año 2015 (Gallagher y Myers, 2014). Se contabilizarían aproximadamente once créditos internacionales, los cuales han sido adquiridos con entidades como el Banco de Desarrollo de China y China Ex Im Bank, en distintos sectores como energía, infraestructura y otros (Gallagher y Myers, 2014).

\section{Conclusiones}

El posicionamiento económico mundial logrado por China durante los últimos años, ha sido un elemento determinante en su relación con América Latina. La tendencia de muchos países latinoamericanos por insertar sus economías en nuevos mercados internacionales y la necesidad de diversificar sus mercados, ha conllevado un notable aumento en las exportaciones de la región hacia China como primer destino comercial. Tal es el caso de Brasil, donde China ha logrado consolidarse como un actor estratégico para su política exterior en distintos niveles.
Por ejemplo, en el afianzamiento de sus relaciones políticas y que ha incidido en la orientación de su política comercial para promover mayores exportaciones hacia el gigante asiático así como en la importancia que representa China en términos de financiamiento internacional para este país del cono sur relativamente industrializado. Asimismo, el bloque BRICS constituye una de las plataformas con mayor proyección en cuanto a las perspectivas de crecimiento económico y comercial que ambos países pueden alcanzar en el nivel internacional. En el caso de Ecuador, como país pequeño andino, ha sido posible observar que durante el mandato del presidente Rafael Correa, China se ha convertido en un actor importante en unos ámbitos más que en otros. En este marco, el Plan Nacional para el Buen Vivir (2013-2017), ha declarado que una de las razones para afianzar relaciones con China radica en la necesidad de generar vínculos con nuevos actores que sean estratégicos para sus intereses. Así China, y su importancia económica en el contexto mundial, constituyen un elemento clave para comprender su importancia dentro de la política exterior del Ecuador. Para ilustrar este punto, China se encuentra entre los principales veinte destinos de exportaciones de mercancías del Ecuador y ocupa un lugar importante en términos de sus exportaciones comerciales. 
No obstante, un tema transversal es el distanciamiento de Ecuador respecto de las fuentes tradicionales de financiamiento como el Banco Mundial y acercamiento a entidades de financiamiento chinas, donde durante los últimos años, Ecuador ha contraído una deuda internacional alta con China en relación al tamaño de su economía. Frente a este escenario, la dependencia financiera subyace como el elemento de peso en la política exterior de un país pequeño como Ecuador respecto a China.

\section{Referencias}

Alianza País. (2006). Manifiesto ideológico de Alianza Pais. Recuperado de http://www.alianzapais.com. ec/2015/07/03/manifiestoideologico.

Basabe-Serrano, S. y Martínez, J. (2014). Ecuador: Cada vez menos democracia, cada vez más autoritarismo...con elecciones, Revista de Ciencia Política, 34(1), pp.145-170.

Comisión Económica para América Latina y el Caribe, CEPAL. (2014). Panorama Social de América Latina 2014. Recuperado de http://repositorio.cepal.org/bitstream/handle/11362/37626/ S1420729_es.pdf
Consejo Empresarial Brasil-China, CEBC. (2015). China Update Brasil. Recuperado de http:// www.cebc.org.br/sites/default/files/china-brazil_update_v.final_oficial.pdf.

Duarte, R. y Trindade, M. (2008). Política exterior brasileña: Nuevos y viejos caminos en los aspectos institucionales, en la práctica del multilateralismo y en la política para el Sur, Revista de Ciencia Política, 28(2), pp.77-106.

El Universo. (2004). Empresas de Brasil cierran 15 acuerdos comerciales con China. Recuperado de http://www.eluniverso. com/2004/05/24/0001/14/ E 9 B 0819370 B B 46 A 7 B. 63B08235668B6C9.html

Gallagher, K. y Myers, M. (2014). China-Latin America Finance Database. Recuperado de http://www. thedialogue.org/map_list/

Grasa, R. (2004). La política exterior de Brasil durante el primer año de presidencia de Lula: un marco para el análisis futuro, Revista CIDOB d'Afers Internacionals, (65, pp.95-109). Recuperado de:https://www.cia.gov/library/publications/resources/ the-world-factbook/

Instituto de Promoción de Exportaciones e Inversiones. (dic 2015/ ene 2016). Análisis de saldos de 
la balanza comercial por socio comercial. En Boletin Mensual de Comercio Exterior, (pp. 1-26).

Instituto de Promoción de Exportaciones e Inversiones. (junio 2014). Evolución de las exportaciones ecuatorianas: enero-abril 2012-2014. Recuperado de http://www.comercioexterior. gob.ec/wp-

content/uploads/downloads/2014/07/ ENE-ABR-2013-2014-paginaweb.pdf

Instituto de Promoción de Exportaciones e Inversiones. (nov/dic 2013). Ecuador: Saldos de Balanza Comercial No Petrolera con Principales Socios. En Boletín Mensual de Comercio Exterior, (11, pp.1-24).

Mansilla, R. (marzo 2012). Asimetrías y convergencias en las relaciones Brasil-China (2003-2011). En X. Ríos (Director), II Simposio Electrónico Internacional sobre Política China 2012. Simposio dirigido por el $\mathrm{Ob}$ servatorio de la Política China, Beijing. Recuperado de http:// www.asiared.com/es/notices/2012/03/ii-simposio-electronico-internacional-sobre-politica-china-2708.php

Organización Mundial del Comercio. (2014). Comercio de Mercancías. En Estadísticas del Comercio
Internacional 2014. Recuperado de https://www.wto.org/spanish/res_s/statis_s/its2014_s/ its14_toc_s.htm

Organización Mundial del Comercio. (2015). Principales economías comerciantes. En Estadísticas del Comercio Internacional 2015. Recuperado de https://www.wto. org/spanish/res_s/statis_s/ its2015_s/its15_toc_s.htm

Pereyra Doval, G. (2013). La autonomía como eje rector de la política exterior. El caso brasileño, Revista Temas y Debates, (25, pp.109-125).

Ramírez, A. (2013). China-América Latina: implicaciones para la hegemonía estadounidense. En J. Orozco y J. Olvera (Coord.), Estados Unidos: ¿Una hegemonía del fin del mundo? (pp. 235-251). México, México: UNAM.

Reformas a la Constitución de la República del Ecuador, Registro Oficial No 449, 20 de octubre de 2008.

Ripley, B. (2002). China: Its Role in the Global Community. En R. Beasley, J. Kaarbo, J. Lantis y M. Snarr (Eds.). Foreing Policy in Comparative Perspective: Domestic and International Influences on State Behavior (pp. 1-21). Washington D.C, Estados Unidos de América: CQ Press. 
Rodríguez, I. (2015). Tendencias de las relaciones de América Latina y el Caribe con la República Popular de China: Un balance reciente 2013-2014. En Adrian B. y Paz M. (Eds.), China en América Latina y el Caribe: Escenarios estratégicos subregionales, (pp.107-124). San José: FLAC$\mathrm{SO}, \mathrm{CAF}$.

Rosales, O. y Kuwayama, M. (2012). China y América Latina y el Caribe: Hacia una relación económica y comercial estratégica. Santiago: Comisión Económica para América Latina y el Caribe.

Secretaría Nacional de Planificación y Desarrollo, SENPLADES (2013). Plan Nacional del Buen Vivir 2013. 2017. Recuperado de http:// www.buenvivir.gob.ec

Silva, Luiz Inácio Lula da. (2004). Pronunciamento do presidente Lula em cadeia nacional 20/05/2004 Página Internacional. Boletim Eletrônico de da Secretaria de Relações Internacionais do Partido dos Trabalhadores, V. Recuperado de www.pt.org.br/site/secretarias_def/secretarias_int_box. asp? $\operatorname{cod}=739 \&$ cod_sis $=9 \&$ ca$\mathrm{t}=9 \mathrm{ol}$

Slipak, A. (2014). América Latina y China: ¿cooperación Sur-Sur o "Consenso de Beijing»?, Nueva Sociedad, (250, pp. 101-113).
Spitaels, G. (2009). China-USA: ¿Dos potencias en conflicto? Madrid, España: Editorial Popular.

Svampa, M. (2013). Consenso de los Commodities y lenguajes de valoración en América Latina, Nueva Sociedad, (244, pp.30-46).

United Nations Conference on Trade and Development, UNCTADSTAT. (2016). Country profile: Brasil. Recuperado de http://unctadstat.unctad.org/ CountryProfile/GeneralProfile/en-GB/076/index.html

United Nations Conference on Trade and Development, UNCTADSTAT. (2016). Country profile: China. Recuperado de http://unctadstat.unctad.org/ CountryProfile/GeneralProfile/en-GB/156/index.html

United Nations Conference on Trade and Development, UNCTADSTAT. (2016). Country profile: Ecuador. Recuperado de http://unctadstat.unctad.org/ CountryProfile/GeneralProfile/en-GB/218/index.html

Xiaoping, S. (2015). China y América Latina en un mundo en transformación: una visión desde China En Adrian B. y Paz M. (Eds.), China en América Latina y el Caribe: Escenarios estratégicos subregionales, (pp.51-73). San José: FLACSO, CAF. 